\title{
Nanoparticulate delivery system for insulin: Design, characterization and in vitro/in vivo bioactivity
}

\author{
Catarina Pinto Reis ${ }^{a, *}$, António J. Ribeiro ${ }^{b}$, Simone Houng ${ }^{c}$, \\ Francisco Veiga ${ }^{a}$, Ronald J. Neufeld ${ }^{c}$ \\ a Laboratory of Pharmaceutical Technology, Faculty of Pharmacy, University of Coimbra, Rua do Norte, 3000-295 Coimbra, Portugal \\ b Laboratory of Pharmaceutical Technology, North Health Sciences Superior Institute, Gandra, Paredes, Portugal \\ c Department of Chemical Engineering, Dupuis Hall, Queen's University, Kingston, Ontario, Canada K7L 3N6
}

\section{A R T I C L E I N F O}

\section{Article history:}

Received 29 October 2006

Received in revised form

21 December 2006

Accepted 29 December 2006

Published on line 13 January 2007

Keywords:

Alginate-dextran nanospheres

Nanospheres

Insulin bioactivity

\begin{abstract}
A B S T R A C T
Insulin-loaded alginate-dextran nanospheres were prepared by nanoemulsion dispersion followed by triggered in situ gelation. Nanospheres were characterized for mean size and distribution by laser diffraction spectroscopy and for shape by transmission electron microscopy. Insulin encapsulation efficiency and in vitro release were determined by Bradford protein assay and bioactivity determined in vitro using a newly developed Western blot immunoassay and in vivo using Wistar diabetic rats. Nanospheres ranged from $267 \mathrm{~nm}$ to $2.76 \mu \mathrm{m}$ in diameter and demonstrated a unimodal size distribution. Insulin encapsulation efficiency was $82.5 \%$. Alginate-dextran particles suppressed insulin release in acidic media and promoted a sustained release at near neutral conditions. Nanoencapsulated insulin was bioactive, demonstrated through both in vivo and in vitro bioassays
\end{abstract}

C) 2007 Elsevier B.V. All rights reserved.
1.

\section{Introduction}

Insulin instability has been regarded as a major obstacle to the development of an insulin oral dosage device aimed at attaining optimal diabetic control. A promising strategy is the use of multifunctional polymers exhibiting gastrointestinal (GI) permeation enhancing and mucoadhesive properties. Interest in using natural materials as part of drug delivery protocols has increased in the past two decades. Alginates are naturally occurring polymers and are known to form a reticulated structure when in contact with calcium ions. This characteristic has been used to produce sustained release particulate systems for a variety of drugs, proteins and even cells (Gombotz and Wee, 1998).

The aim of this study was to prepare and characterize an insulin-loaded alginate-dextran nanopolymeric delivery sys- tem. A drug carrier for insulin should provide a stable and biocompatible environment to ensure that the main fraction of the therapeutic protein will be biologically active following encapsulation. The carrier should stabilize and preserve physiological activity during both particle processing and insulin release. Once absorbed through the epithelial cell layer (intestinal barrier), released or particulate insulin can interact with cell-surface receptors or be captured by lymphatic cells, or pass through or be entrapment in the lymph nodes or transfer to the blood (Damgé et al., 2007).

An alginate nanopolymeric system is proposed through nanoemulsion dispersion of alginate/insulin solution, followed by triggered in situ gelation through instantaneous release of $\mathrm{Ca}^{2+}$ from insoluble complex. This technique has been applied to the formulation of macro- and microspheres (Vandenberg and Nouè, 2001), but has not been extended or

\footnotetext{
* Corresponding author. Tel.: +351 239855085; fax: +351 239855099.

E-mail address: catarinareis@ci.uc.pt (C.P. Reis). 
demonstrated at the nano-scale. Particles are formed under mild conditions, using methods which are rapid and readily scaleable with little manipulation (Reis et al., 2006a). However, shear stress during homogenization and centrifugation, and protein adsorption onto matrix polymer, and surface tension effects may lead to denaturation, molecular breakdown, fibrillation and protein aggregation (De Rosa et al., 2000). Thus it is important to test the biological activity of insulin following encapsulation.

Biological insulin activity cannot be quantified from the insulin molecular structure itself. Nevertheless, insulin action may be characterized by binding at the cell surface receptor inducing phosphorylation of the kinase receptor followed by a cascade of signaling protein activations. One such protein is Akt, a downstream effector of the insulin signaling pathway (Alessi and Downes, 1998; Elghazi et al., 2006). Antibodies to activated insulin receptor itself are highly non-specific and therefore not reliable. However, it is possible to relate activation status of cell signaling proteins further in the insulin activation pathway to activity of the insulin molecule. The antibody for phosphorylated Akt is very specific, with a single band appearing on Western blots (Patel et al., 2001), providing a strong and reproducible signal. Quantification of bioactive insulin following encapsulation and release, was demonstrated using the developed Western blot immunoassay, and through monitoring blood glucose levels following subcutaneous administration of released insulin, in diabetic rats.

Nanospheres were characterized in terms of size distribution, morphology, encapsulation efficiency, in vitro insulin release behaviour and biological activity of insulin following encapsulation.

\section{Materials and methods}

\subsection{Materials}

Sodium alginate (supplier's specifications: viscosity of $2 \%$ solution at $25^{\circ} \mathrm{C}, 250 \mathrm{cps}$ ) and dextran sulfate (approximately MW $5 \mathrm{kDa}$ ) were purchased from Sigma Chemical Co. (St. Louis, MO, USA). Setacarb 06 calcium carbonate was obtained from Omya (Orgon, France). Paraffin oil was supplied by Vaz Pereira (Lisbon, Portugal). The emulsifier, Span 80, was purchased from Fluka, Chemie $\mathrm{GmbH}$ (Buchs, Switzerland). Insulin was kindly donated by Hospitais da Universidade de Coimbra (Actrapid Insulin ${ }^{\circledR}$ from Novo Nordisk, Bagsvaerd, Denmark). Streptozotocin was purchased from Sigma (Steinheim, Germany). All other chemicals were of reagent grade or equivalent.

\subsection{Preparation of alginate-dextran nanospheres}

The preparation method for alginate-dextran nanospheres was adapted from the emulsification/internal gelation technique described previously (Poncelet et al., 1992). By controlling the conditions under which the water-in-oil emulsion is produced, dispersed droplet size and thus resulting particle size can be controlled. It was the intent of this study to extend the range of particle sizes from that of macro- and microspheres, to that in the nanometer size range. An aque- ous solution of low viscosity sodium alginate $(2 \%, w / v)$ and dextran sulfate $(0.75 \%, \mathrm{w} / \mathrm{v})$ was prepared by suspending the polymer and adjuvant, respectively, in distilled water followed by overnight stirring on an orbital shaker (100 rpm). Low viscosity sodium alginate was used to obtain small particles. Insulin $(100 \mathrm{IU} / \mathrm{mL}, 10 \mathrm{~mL})$ was mixed into alginate-dextran solution and the resulting solution held stationary for at least $1 \mathrm{~h}$ to allow deaeration. An aqueous suspension of ultrafine calcium carbonate $(5 \%, \mathrm{w} / \mathrm{v})$ was sonicated for $30 \mathrm{~min}$ to break up crystal aggregates, and then dispersed into the alginate-dextran-insulin solution (calcium-alginate ratio, $7 \%$, $\mathrm{w} / \mathrm{w})$. The resulting mixture was emulsified within paraffin oil aided by Span $80(1.5 \%$, v/v) using a mixing impeller at $1600 \mathrm{rpm}$. After $15 \mathrm{~min}$ emulsification, gelation was triggered by addition of $20 \mathrm{~mL}$ paraffin oil containing glacial acetic acid (acid-calcium molar ratio, 3). The intent was to reduce the $\mathrm{pH}$ of the alginate-calcium solution from 7.8 to 4.5 , releasing soluble calcium from carbonate complex. After $60 \mathrm{~min}$, an acetate buffer solution $(70 \mathrm{~mL}$ at $\mathrm{pH} 4.5$, United States Pharmacopeia, USP XXVIII) with dehydrating solvents (acetone, isopropanol and hexane, $15 \mathrm{~mL}: 10 \mathrm{~mL}: 5 \mathrm{~mL}$, respectively) was added to the oil-particle suspension and nanospheres were recovered by centrifugation $(12,500 \times g$ during $10 \mathrm{~min})$. Nanospheres were separated in two fractions. One fraction of nanospheres to be used for particle characterization was stored in acetate buffer at $\mathrm{pH} 4.5$, referred to as hydrated nanospheres. A second fraction was frozen in an ethanol bath at $-50^{\circ} \mathrm{C}$ and lyophilized (Lyph-lock 6, Labconco, Kansas City, MS, USA) at $0^{\circ} \mathrm{C}$ for $48 \mathrm{~h}$ and stored at $4{ }^{\circ} \mathrm{C}$. Insulin-free nanospheres were prepared as controls.

\subsection{Characterization of nanospheres}

Size distribution analysis was performed by laser diffraction spectrometry using a Coulter LS130 granulometer (Beckman Coulter Inc., Fullerton, CA). Mean diameters of aqueous suspensions were determined in triplicate and size distribution was represented by number.

Morphology was assessed by transmission electron microscopy (Zeiss EM 902A, Germany) by placing samples onto carbon grids, negative staining with uranyl acetate and drying at room temperature.

\subsection{Determination of encapsulation efficiency}

The encapsulation efficiency was measured by incubating $30 \mathrm{mg}$ nanospheres in sodium citrate $(55 \mathrm{mM}) /$ phosphate buffer at pH 7.4 (USPXXVIII) for $1 \mathrm{~h}$ (100 rpm). After particle dissolution, the mixture was centrifuged and protein content in supernatant analyzed by Bradford method (Coomassie Protein Assay Reagent from Pierce, USA) at $595 \mathrm{~nm}$. Insulin-free nanospheres were used as control. Drug content was quantified and encapsulation efficiency (\%) determined by insulin released as percentage of initial amount used in formulation.

\subsection{In vitro release of insulin under simulated gastrointestinal conditions}

Drug release studies were focused on the release behaviour at gastric and intestinal $\mathrm{pH}$ conditions. Lyophilized insulin- 
loaded nanospheres $(10 \mathrm{mg})$ were incubated in $10 \mathrm{~mL}$ hydrochloric acid buffer at $\mathrm{pH} 1.2$ (USP XXVIII), under continuous magnetic stirring (100 rpm, $2 \mathrm{~h}$ ) at $37^{\circ} \mathrm{C}$. Samples $(1.5 \mathrm{~mL})$ at appropriate intervals were withdrawn and assayed for protein $(1 \mathrm{~mL})$. Fresh dissolution medium was added to maintain a constant volume. To simulate the progress of nanospheres moving from the stomach into the upper small intestine, the buffer was changed after $2 \mathrm{~h}$ to higher $\mathrm{pH}$. Nanospheres were centrifuged $(12,500 \times \mathrm{g}, 10 \mathrm{~min})$ then re-suspended into $10 \mathrm{~mL}$ phosphate buffer at pH 6.8 (USP XXVIII), under continuous magnetic stirring $(100 \mathrm{rpm})$ during $6 \mathrm{~h}$. Supernatant samples at appropriate intervals were withdrawn and assayed for protein and fresh dissolution medium added to maintain a constant volume. Insulin-free nanospheres served as negative control. Experiments were performed in triplicate and cumulative insulin release expressed as percentage of initial insulin loading.

\subsection{In vitro and in vivo bioassay}

Nanoencapsulated and released insulin was assayed for bioactivity in vitro by developing a Western blotting technique to detect the phosphorylation status of a downstream effector of the insulin signaling pathway, Akt (Patel et al., 2001). Bioassay was also conducted in vivo by subcutaneous injection of released insulin to diabetic rats. Released insulin was dispersed into phosphate buffered saline (PBS, pH 7.4), centrifuged and the supernatant used for in vivo and in vitro bioassays.

Rat L6 myoblasts were cultured in Dulbecco's Modified Eagle's Medium (DMEM) containing 10\% fetal bovine serum, $1 \%$ glutamine and 1\% antimycotic (Gibco, Invitrogen Corporation) on $35 \mathrm{~mm}$ culture dishes. Cells were stimulated with non-encapsulated insulin and with insulin recovered from nanospheres for different time periods. After stimulation, cells were lysed with RIPA lysis buffer $(1 \%$, w/w) and centrifuged at $4{ }^{\circ} \mathrm{C}$ (Eppendorf 5417R, 20,000 $\times$ g, $15 \mathrm{~min}$ ). Lysates were analyzed by Bradford protein assay. Samples were subsequently analyzed by Western blot for $\alpha$-Akt (Cell Signaling Technology, 1:1000), $\alpha$-phospho-Akt (Ser473) (Cell Signaling Technology and New England BioLabs, 1:2000), $\alpha-$ actin (Santa Cruz Biotechnology, 1:500), and $\alpha$-pY99 (Santa Cruz Biotechnology, 1:2000). Actin and pY99 antibodies were used as controls. Immunoblots were developed in a Kodak M35A X-OMAT processor and exposed to Kodak X-OMAT Blue XB-1 film. Western blot results were scanned as black and white transmissive photos at $300 \mathrm{dpi}$ using VistaScan software. They were then analyzed by densitometry using Corel Photo-Paint 11 software. The ratio of intensities of phosphoAkt/Akt was observed from regression analysis and dilution factor, and the insulin activity was determined. The percent recovery of activity was determined from difference between predicted and measured activity as percentage of predicted.

Male Wistar rats weighing about $250 \mathrm{~g}$ were housed in a 12-12-h light-dark cycle and constant temperature environment of $22^{\circ} \mathrm{C}$. All animal procedures were reviewed and approved by the committee for animal research according the Portuguese Law (DL no. 197/96) and the Institutional European Guidelines (no. 86/609).
Table 1 - Different treatments used for in vivo

experiments

Animal group

Treatment

\begin{tabular}{ll}
\hline I & Insulin-free nanospheres \\
II & Dispersion medium (PBS) \\
III & Fasting (no treatment) \\
IV & Non-encapsulated insulin at $1 \mathrm{IU} / \mathrm{kg}$ \\
V & Non-encapsulated insulin at $4 \mathrm{IU} / \mathrm{kg}$ \\
VI & Insulin released from nanospheres at $1 \mathrm{IU} / \mathrm{kg}$ \\
VII & Insulin released from nanospheres at $4 \mathrm{IU} / \mathrm{kg}$
\end{tabular}

Diabetes was induced with single intraperitoneal injection of $50 \mathrm{mg} / \mathrm{kg}$ streptozotocin in citrate buffer at $\mathrm{pH} 4.5$ damaging pancreatic $\beta$-cells without reducing exocrine functions. During the first $24 \mathrm{~h}$, rats were given $5 \%$ glucose to prevent hypoglycemia due to destruction of pancreatic $\beta$-cells. After 8-10 days of the streptozotocin treatment, rats with frequent urination, loss of weight, and blood glucose levels higher than $250 \mathrm{mg} / \mathrm{dL}$ were selected and randomly divided into seven groups as outlined in Table 1. Before testing, animals were fasted overnight with free access to water. To minimize the diurnal blood glucose fluctuations, experiments were performed in the morning (Hovgaard et al., 1996).

Insulin-loaded nanospheres were suspended in $20 \mathrm{~mL}$ PBS at $20^{\circ} \mathrm{C}$ for $2 \mathrm{~h}$ under magnetic stirring $(100 \mathrm{rpm})$ to extract insulin, and supernatant protein quantified. Samples were passed under vacuum through a $0.45 \mu \mathrm{m}$ filter, centrifuged $(12,500 \times g, 10 \mathrm{~min})$, re-filtered, and the supernatant was re-assayed for insulin content. Suspension medium and insulin-free nanospheres were treated in the same manner and the supernatants were used as negative controls. Fasting effect was also assayed as negative control. Released insulin in PBS was injected subcutaneously at doses of 1 and $4 \mathrm{IU} / \mathrm{kg}$. Non-encapsulated insulin was injected at the same doses and used as positive controls. Blood samples were taken from the tip of the tail vein and blood glucose levels measured using a Medisense Precision Xtra glucometer (Abbot, USA).

Data are presented as means \pm standard error of mean (S.E.M.). Statistical evaluation was performed with a one-way ANOVA followed by a Dunnett multiple comparison test. A $P<0.05$ was taken as the criterion of significance.

The areas under the reduction of blood glucose concentration versus time after injection were calculated by using the linear trapezoidal rule.

\section{Results and discussion}

\subsection{Nanosphere characterization}

Nanospheres were prepared by emulsion dispersion/in situ triggered gelation resulting in an unimodal size distribution as seen in Fig. 1. Approximately $90 \%$ of the particles had a diameter of less than $1 \mu \mathrm{m}\left(\mathrm{D}_{90}\right)$ and $50 \%$ less than $564 \mathrm{~nm}$ $\left(D_{50}\right)$. Particle size was lower than the critical size necessary to enable GI absorption by M-cells on Peyer's patches (Norris et al., 1998; Saez et al., 2000). Some studies cite $5 \times 10^{3} \mathrm{~nm}$ while others describe particles well under $10 \times 10^{3} \mathrm{~nm}$ as critical for absorption (Eldridge et al., 1990). As seen in Fig. 2, some nanoaggregates were observed but most nanospheres 


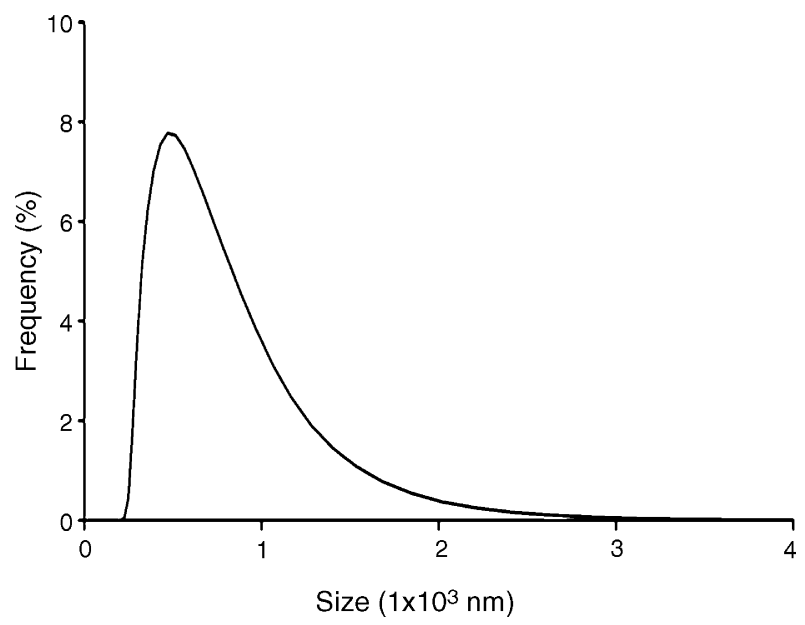

Fig. 1 - Particle size distribution of insulin nanospheres in terms of number.

appeared spherical and discrete. Additional factors contribute to the nS intestinal uptake, including polymer characteristics such as hydrophobicity, bulk properties and charge (Norris et al., 1998).

\subsection{Encapsulation efficiency}

Encapsulation efficiency was $82.5 \pm 3.3 \%$ of the initial amount of insulin formulated. $\mathrm{pH}$ can have an important affect on encapsulation efficiency. As insulin has an isoelectric point around 5.3 (Chien, 1996) and alginate has pK values of 3.38 and 3.65 for $\mathrm{M}$ and $\mathrm{G}$ residues, respectively (Draget et al., 1994), electrostatic attractions at the final alginate $\mathrm{pH} 4.5$ may occur providing high encapsulation efficiency of the oppositely charged protein.

\subsection{In vitro release of insulin under simulated gastrointestinal conditions}

Insulin behaviour through the gastrointestinal tract was simulated in vitro in order to determine if the insulin is being

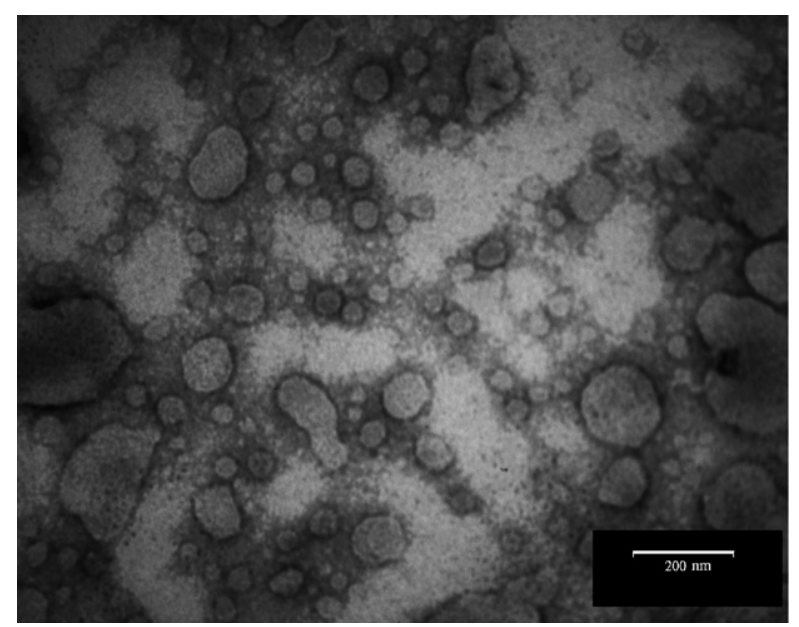

Fig. 2 - Photograph obtained by transmission electron microscopy of insulin nanospheres.

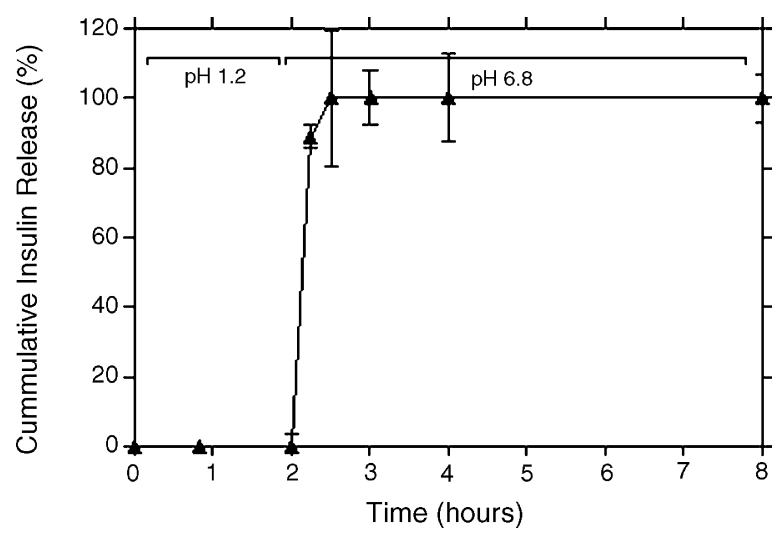

Fig. 3 - Release behaviour of insulin alginate-dextran nanospheres under physiological conditions. Each value represents mean \pm S.D. $(n=3)$.

released prematurely in the stomach or if there is insulin to be absorbed in the intestine. Fig. 3 depicts release profiles of insulin from nanospheres under simulated gastrointestinal conditions. At low gastric $\mathrm{pH}$, insulin was fully retained likely due to alginate polymer forming a compact acid-gel structure reducing permeability and potentially stabilizing insulin from acid attack. Up to $89 \%$ of the insulin was released almost immediately after changing the medium to near neutral $\mathrm{pH}$, and full release was observed after $1 \mathrm{~h}$. The extent of release in vivo was not determined but it appears that the gel swells, potentially releasing some of the insulin, as the in vitro assay was optimized to promote release, in contrast to in vivo conditions. Insulin then may be released into the GI tract, and some may be retained by nanoparticles. $\mathrm{pH}$-dependent insulin release as well as high alginate porosity has a significant influence on the insulin release profile.

Drug release from alginate matrices may be modulated by a dissolution-erosion process. At low $\mathrm{pH}$, alginates contract due to alginic acid precipitation as previously described (Almeida and Almeida, 2004), resulting in a compact and impermeable polymer matrix, retaining insulin, but potentially excluding proteases. $\mathrm{Ca}^{2+}$ is released from the acidic gel, potentially destabilizing the gel at subsequent neutral $\mathrm{pH}$. The alginate matrix then swells, promoting drug release. Swelling is further enhanced by the presence of phosphate ion $\mathrm{PO}_{4}{ }^{3-}$ and by counter-ions such as $\mathrm{Na}^{+}$(Ramdas et al., 1999). As well, at neutral $\mathrm{pH}$, both alginate and insulin are negatively charged and electrostatic repulsion may promote insulin release. Insulin is rapidly released from nanospheres once in neutral conditions determined through in vitro conditions, optimized to promote release. The extension to insulin release in vivo is not clear, but it appears that the gel swells, potentially releasing some of the insulin. However, retained insulin would then be absorbed in a nanoparticulate form.

\subsection{In vitro and in vivo bioassay}

An in vitro bioassay was developed for detection of protein kinase B (Akt or PKB), activated by phosphorylation in response to insulin binding to cell surface receptors. The assay utilized rat $\mathrm{L} 6$ myoblasts stimulated with insulin and ana- 
Table 2 - Percentage recovered activity of alginate nanospheres

\begin{tabular}{lc} 
Sample & Formulation \\
\hline Ratio of intensities of phosphoAkt/Akt ${ }^{\mathrm{a}}$ & 0.299 \\
Activity (nM) & 1.25 \\
Predicted activity (nmol/mg) & 2.28 \\
Recovery activity (\%) & 55 \\
\hline & \\
a Ratio of intensities of phosphoAkt/Akt $=0.0346 \mathrm{e}^{0.0346 \text { concentration }}$ \\
plus dilution factor. \\
$\begin{array}{l}\text { b Intensities of } 0.1 \mathrm{mg} \text { stimulation, relative to } \\
\text { encapsulated insulin. }\end{array}$ \\
\hline
\end{tabular}

lyzed by Western immunoblot detecting Akt phosphorylation conducted on cell lysates. Time course and concentration studies determined a suitable stimulation of $10 \mathrm{~min}$ and $100 \mathrm{nM}$ insulin for quantitation. Akt phosphorylation was not observed prior to insulin stimulation, and slight stimulation detected following 1 and 2 min post-stimulation. Standard curves were linear and increased with increasing concentrations of insulin, with a minimum detection limit of $2 \%$ of the $100 \mathrm{nM}$ insulin signal. This linear relationship suggests that the assay is a suitable method by which insulin bioactivity can be established. Subsequent assays on insulin released from nanospheres can be quantified relative to $100 \mathrm{nM}$ insulin stimulation. Activity levels with stimulations equivalent to $0.1 \mathrm{mg}$ of nanospheres are summarized in Table 2. Bioactivity was $55 \%$ of the theoretical value, showing an important fraction of the released insulin was biologically active.

An in vivo bioassay was conducted by subcutaneously injecting insulin extracted from nanospheres into diabetic rats. Results in Fig. 4 indicate an equivalent hypoglycemic effect with significant glycemia decrease for all insulin solutions. The glycemic profiles indicate that all the insulin forms display similar biological and biopharmaceutical behaviour

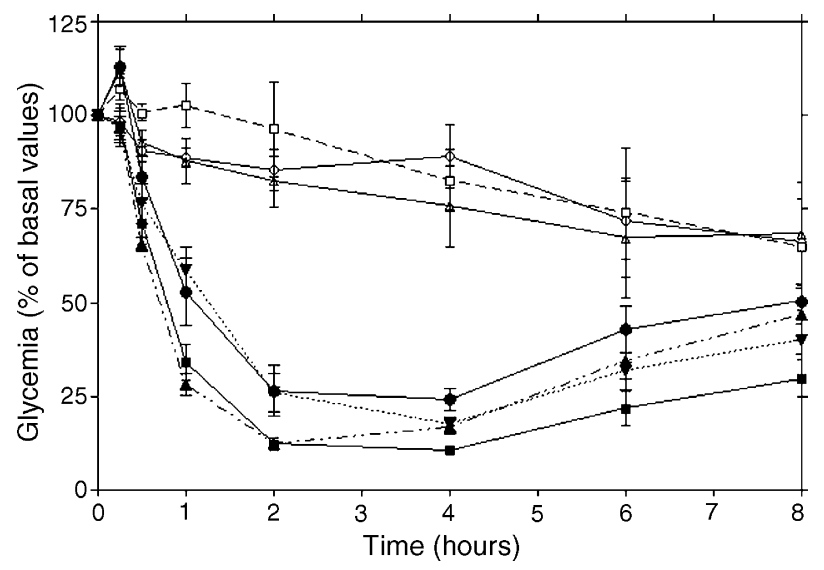

Fig. 4 - Blood glucose levels of SZT-induced diabetic rats after injection of tested formulations: $(\Delta)$ free-insulin nanospheres, $(\square)$ suspension medium (phosphate buffered saline), $(O)$ untreated, $(\nabla)$ non-encapsulated insulin at $1 \mathrm{IU} / \mathrm{kg}$, ( $\Lambda$ ) non-encapsulated insulin at $4 \mathrm{IU} / \mathrm{kg},(0)$ insulin released from nanospheres at $1 \mathrm{IU} / \mathrm{kg}$, and finally ( $\square$ ) insulin released from nanospheres at $4 \mathrm{IU} / \mathrm{kg}$. Each data point represents the mean \pm S.E.M., from $n=6$.

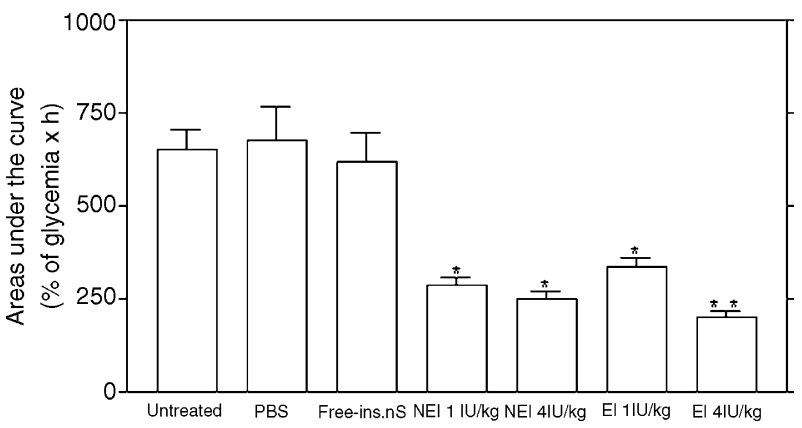

Fig. 5 - Area under curve demonstrating the hypoglycaemic properties of insulin released from nanospheres: untreated (WT), suspension medium (phosphate buffered saline, PBS), free-insulin nanospheres, non-encapsulated insulin at 1 and $4 \mathrm{IU} / \mathrm{kg}$ (NEI) and finally, insulin encapsulated and then released from nanospheres at 1 and $4 \mathrm{IU} / \mathrm{kg}$ (EI). Each data point represents the mea \pm S.E.M. from $n=6$. "Significant difference from WT: $P<0.001$ and ** significant difference from non-encapsulated insulin: $P<0.0535$.

either in terms of maximal activity or pharmacological time course. The hypoglycemic action of both insulin released from nanospheres and insulin control was rapid with onset time of $0.5-1 \mathrm{~h}$ reaching maximal activity within $2-4 \mathrm{~h}$. The time of glucose depression lasted for $8 \mathrm{~h}$. Basal blood glucose levels decreased 76 and $89 \%$ within $4 \mathrm{~h}$ for insulin released from nanospheres at 1 and $4 \mathrm{IU} / \mathrm{kg}$, respectively. Neither blank nanospheres in PBS or PBS alone showed any biological effect. As illustrated in Fig. 5, all insulin formulations (released and controls) significantly decreased blood glucose levels with statistical differences compared to the non-treated group $(P<0.001)$. Area under the blood glucose curve, decreased with an increase of insulin dose as shown in Fig. 5. In addition, insulin released from nanospheres at $4 \mathrm{IU} / \mathrm{kg}$ demonstrated statistical differences in comparison to non-encapsulated insulin $(P<0.054)$ possibly due to the filtration step. Before dosing, all formulations were filtered through a $0.45 \mu \mathrm{m}$ membrane. Particle sizes varied from $267 \mathrm{~nm}$ to $2.76 \mu \mathrm{m}$ which means that some particles may have been collected with released insulin and injected simultaneously. It is also possible that insulin is strongly associated with alginate and consequently is not immediately released from the alginate matrix. These two reasons may explain the lasting hypoglycemic effect of insulin released at $4 \mathrm{IU} / \mathrm{kg}$.

It was previously reported that emulsion-based encapsulation methods may promote protein unfolding due to mechanical shear stress and the oil-water interface (Putney, 1998). As well, acid pH used to solubilize calcium salt during particle manufacture combined with agitation, can also facilitate dissociation of insulin tetramers/dimers into monomers, and to further cause the monomer to adopt a partially unfolded intermediate conformation (Ma et al., 2002). None of these factors led to protein secondary structure modification, as previously demonstrated by circular dichroism and by HPLC-RP/HLPC-mass (Reis et al., 2006b). Several factors may contribute to this stabilization and explain previous results. 
When emulsion-dispersions were prepared, the total interfacial area increased as the droplet size decreased. Surfactants may provide protection from exposure to the oil-water interface mainly due to two reasons. One is due to a steric effect which blocks aggregation-prone hydrophobic sites on the protein surface (Bam et al., 1998) and the other due to a competitive effect with the protein for space at the surface, thereby avoiding part of the protein from reaching the interface, which would expose insulin to the oil-water interface and subsequent adsorption and structural damage. Additionally, the probability of dissociation of insulin tetramers/dimers into monomers was avoided since greatly diluted acid suspensions combined with agitation were used during encapsulation to trigger gelation of the alginate polymer. Finally, this emulsification methodology was performed under mild conditions maintaining insulin biological activity.

Presently, studies are underway on the gastrointestinal uptake and activity of orally dosed insulin nanospheres.

\section{Conclusions}

Nanospheres obtained by emulsion dispersion/in situ triggered gelation of alginate polymer, may have potential for an oral dosage system for insulin and potentially other protein-based drugs. Insulin-nanospheres demonstrated an incorporation efficiency of $80 \%$ and unimodal size distribution. A new in vitro assay to measure the biological activity of insulin was described and subcutaneous administration of released insulin to diabetic rats demonstrated that bioactivity was largely retained in the peptide-loaded nanospheres. Nanospheres were able to protect and preserve protein stability during particle formulation, recovery and insulin release in PBS.

\section{Acknowledgments}

The authors are grateful for financial support from the Fundação para a Ciência e Tecnologia, Portugal (SFRH/BD/13673/ 2003) and from the Natural Sciences and Engineering Research Council of Canada. The authors would like to thank to Dr. Isabel Vitória Figueiredo for her collaborative help in vivo studies and Dr. Peter Greer and Michelle Scott of the Queen's University Cancer Research Institute for their remarkable support in helping develop the in vitro bioassay.

\section{REFERENCES}

Alessi, D.R., Downes, C.P., 1998. The role of PI 3-kinase in insulin action. Biochim. Biophys. Acta (BBA), Mol. Cell Biol. Lipids 1436, 151-164.

Almeida, P.F., Almeida, A.J., 2004. Cross-linked alginate-gelatine beads: a new matrix for controlled release of pindolol. J. Control. Rel. 97, 431-439.
Bam, N.B., Cleland, J.L., Yang, J., Manning, M.C., Carpenter, J.F., Kelley, R.F., Randolph, T.W., 1998. Tween protects recombinant human growth hormone against agitation-induced damage via hydrophobic interactions. J. Pharm. Sci. 87, 1554-1559.

Chien, Y.W., 1996. Human insulin: basic sciences to therapeutic uses. Drug Dev. Ind. Pharm. 22, 753-789.

Damgé, C., Maincent, P., Ubrich, N., 2007. Oral delivery of insulin associated to polymeric nanoparticles in diabetic rats. J. Control. Rel. 117, 163-170.

De Rosa, G., Iommelli, R., La Rotonda, M.I., Miro, A., Quaglia, F., 2000. Influence of the co-encapsulation of different non-ionic surfactants on the properties of PLGA insulin-loaded microspheres. J. Control. Rel. 69, 283-295.

Draget, K.I., Skjåk-Braek, G., Smidsrød, O., 1994. Alginic acid gels: the effect of alginate chemical composition and molecular weight. Carbohyd. Polym. 25, 31-38.

Eldridge, J.H., Hammond, C.J., Meulbroek, J.A., Staas, J.K., Gilley, R.M., Tice, T.R., 1990. Controlled vaccine release in gut-associated lymphoid tissues. I. Orally administered biodegradable microspheres target the peyer's patches. J. Control. Rel. 11, 205-214.

Elghazi, L., Balcazar, N., Bernal-Mizrachi, E., 2006. Emerging role of protein kinase B/Akt signaling in pancreatic [beta]-cell mass and function. Int. J. Biochem. Cell Biol. 38, 157-163.

Gombotz, W.R., Wee, S.F., 1998. Protein release from alginate matrices. Adv. Drug Deliv. Rev. 31, 267-285.

Hovgaard, L., Jacobs, H., Wilson, D.E., Kim, S.W., 1996. Stabilization of insulin by alkylmaltosides. B. Oral absorption in vivo in rats. Int. J. Pharm. 132, 115-121.

Ma, Z., Yeoh, H.H., Lim, L.-Y., 2002. Formulation pH modulates the interaction of insulin with chitosan nanoparticles. J. Pharm. Sci. 91, 1396-1404.

Norris, D.A., Puri, N., Sinko, P.J., 1998. Effect of physical barriers and properties on the oral absorption of particulates. Adv. Drug Deliv. Rev. 34, 135-154.

Patel, N., Craddock, B.L., Staniforth, J.N., Tobyn, M., JWelham, M., 2001. Spray-dried insulin particles retain biological activity in rapid in-vitro assay. J. Pharm. Pharmacol. 53, 1415-1418.

Poncelet, D., Lencki, R., Beaulieu, C., Halle, J.P., Neufeld, R.J., Fournier, A., 1992. Production of alginate beads by emulsification/internal gelation. I. Methodology. Appl. Microbiol. Biotechnol. 38, 39-45.

Putney, S.D., 1998. Encapsulation of proteins for improved delivery. Curr. Opin. Chem. Biol. 2, 548-552.

Ramdas, M., Dileep, K.J., Anitha, Y., Paul, W., Sharma, C.P., 1999. Alginate encapsulated bioadhesive chitosan microspheres for intestinal drug delivery. J. Biomater. Appl. 13, 290-296.

Reis, C.P., Neufeld, R.J., Ribeiro, A.J., Veiga, F., 2006a. Design of insulin-loaded alginate nanoparticles: influence of calcium ion on polymer gel matrix properties. Chem. Ind. Chem. Eng. Quart. 12, 47-52.

Reis, C.P., Ribeiro, A.J., Neufeld, R.J., Veiga, F., 2006b. Alginate microparticles as novel carrier for oral insulin delivery. Biotechnol. Bioeng. 96, 1-13.

Saez, A., Guzman, M., Molpeceres, J., Aberturas, M.R., 2000. Freeze-drying of polycaprolactone and poly(D,L-lactic-glycolic) nanoparticles induce minor particle size changes affecting the oral pharmacokinetics of loaded drugs. Eur. J. Pharm. Biopharm. 50, 379-387.

Vandenberg, G.W., Nouè, J.D.L., 2001. Evaluation of protein release from chitosan-alginate microcapsules produced using external or internal gelation. J. Microencapsulation 18, 433-441. 